\title{
Re: percutaneous nephrostomy for malignant ureteric obstruction: Have we got the balance right?
}

\author{
Nabil K. Bissada • Mohamed H. Kamel
}

Received: 18 April 2013/Accepted: 18 April 2013/Published online: 12 May 2013

(C) Springer Science+Business Media Dordrecht 2013

The authors describe their experience with decompressing the renal collecting system using percutaneous nephrostomy with antegrade stenting to follow in most of their patients. The data are sobering. Nearly half of their patients had complications with one death secondary to PCN insertion. The average survival after PCN insertion was 78 days.

While the experience in the USA may be more favorable following the simple insertion of PCN for MUO, the authors experience help to demonstrate the risks inherent in this procedure in this vulnerable group of patients. Further, it is important to remember that PCN insertion alone does not prolong the life of patient with advanced metastatic disease like the typical group presented in this analysis (55\% of their patients had metastases at presentation).

The main message and remaining question in this study is the decades long dilemma of whether and when to drain the kidney in terminally ill patients secondary to malignancy especially in those with limited life expectancy who will not benefit and in fact

N. K. Bissada $(\bowtie)$

Department of Urology, University of Oklahoma Health

Sciences Center, 920 SL Young, WP3150, Oklahoma

City, OK 73102, USA

e-mail: bissadan@hotmail.com

M. H. Kamel

Department of Urology, University of Arkansas for Medical Sciences, 4301 West Markham \#774, Little

Rock, AR 72205, USA may be harmed by the morbidity and the need for longer hospitalization that consumes significant proportion of their remaining life. Those with prostate cancer and MUO may have slightly longer survival. Needless to say that in each case, the patient and/or the family should be empowered to make a truly wellinformed decision.

In the particular situation of upward stent migration with failure to retrieve it using the ureteroscope, a new PCN usually allows its retrieval in an antegrade manner. It is not clear why an open surgery was required to retrieve the migrated stent in a terminally ill cancer patient.

Bottom line is that the decision for decompressing the collecting system in a terminally ill cancer patient to relieve MUO should be approached very diligently in view of the patient's short life expectancy considering the morbidity and the potential to worsen patient's quality of life.

Conflict of interest The authors declare that they have no conflict of interest. 